\title{
The Diagnostic Value of Sonographic Findings in Axillary Lymphadenopathy
}

\author{
Elif Colak ${ }^{1}$, Gultekin Ozan Kucuk ${ }^{1, ~}$, Kadir Yildirim ${ }^{1}$, Onur Ozturk ${ }^{2}$ \\ ${ }^{1}$ Department of General Surgery, Samsun Education and Research Hospital, Samsun, Turkey \\ ${ }^{2}$ Asarcik Community Health Center, Samsun, Turkey
}

\section{Email address:}

gultekinozan@hotmail.com (G. O. Kucuk)

\section{To cite this article:}

Elif Colak, Gultekin Ozan Kucuk, Kadir Yildirim, Onur Ozturk. The Diagnostic Value of Sonographic Findings in Axillary Lymphadenopathy. Journal of Family Medicine and Health Care. Vol. 1, No. 2, 2015, pp. 21-23. doi: 10.11648/j.jfmhc.20150102.11

\begin{abstract}
Background: Axillary lymphadenopathy (LAP) is one of the most common pathologies encountered in daily practice. The aim of this study is to evaluate the demographic, ultrasonographic, and biopsy findings of patients who underwent an excisional biopsy to distinguish between benign and malignant LAP of the axillary region. Methods: Patients who underwent an excisional biopsy between January 2008 and June 2013 in Samsun Education and Research Hospital were evaluated retrospectively. The exclusion criterion was patients who were diagnosed with breast cancer before admission. According to their pathology results, 23 patients were classified as Group $1(n=4)$ and Group $2(n=19)$ based on the presence of malignant and benign lymph node involvement, respectively. The demographic data (age and gender) and ultrasonographic findings (shape, diameter, and number of lymph nodes) of the two groups were then compared. Results: Seventeen (73.91\%) of the patients were female, and $6(26.09 \%)$ were male. According to the pathology results, a malignant lymphoma was diagnosed in four patients in Group 1. Granulomatous lymphadenitis $(n=6)$ and reactive lymphadenitis $(n=13)$ were diagnosed in Group 2. There was no statistical difference between the groups in terms of age (55 vs 47 years). There was a statistically significant between-group difference in gender, with a male gender predominance in the malignant lymphoma group $(p=0.04)$. In Group 1 , all the patients $(100 \%)$ had multiple enlarged lymph nodes, whereas only $11(57.8 \%)$ of the patients in Group 2 had multiple enlarged lymph nodes. There was no significant difference between the groups in the numbers of lymph nodes $(p=0.15)$. There was statistically significant difference between groups in terms of lymph node diameter ( $45 \mathrm{vs} 26 \mathrm{~mm})(p=0.02)$. Conclusion: Ultrasonography (US) is a valuable tool in the diagnosis of patients with axillary LAP. Our findings suggest that a histopathological examination is warranted in male patients with an axillary LAP larger than $2 \mathrm{~cm}$ determined by US.
\end{abstract}

Keywords: Axilla, Lymphadenopathy, Ultrasonography, Pathology, Lymphoma

\section{Introduction}

Axillary lymphadenopathy (LAP) is one of the most common pathology encountered in the axillary region. LAP is defined as an abnormality in the size and characteristics of the lymph node. It may be the result of inflammatory or neoplastic events caused by systemic diseases or malignancies, such as breast cancer [1].

Axillary masses or accessory breast may mimic pathological lymph nodes [2]. Nodal hyperplasia, collagen tissue diseases, granulomatous diseases, silicone LAP, and infectious diseases are benign causes of LAP of the axillary region. Lymphoproliferative diseases, breast cancer, and metastasis due to malignancies other than breast cancer are usually accepted as malignant causes of axillary LAP. Additional causes of axillary LAP are hematomas, skin lesions, hydraadenitis suppurativa, cystic hygromas, and lymphangiomas [3]. A biopsy procedure is required to distinguish between benign and malignant causes of LAP. Physical and radiological findings may provide clues about which patients should undergo a biopsy.

The aim of this study was to evaluate the demographic, ultrasonographic, and biopsy findings of patients who underwent an excisional biopsy to distinguish between benign and malignant LAP of the axillary region.

\section{Patients and Methods}

Patients who underwent an excisional biopsy between January 2008 and June 2013 in Samsun Education and Research Hospital were evaluated retrospectively. The exclusion criterion was patients who were diagnosed with 
breast cancer before admission. According to their pathology results, 23 patients were classified as Group $1(n=4)$ and Group $2(n=19)$ based on the presence of malignant and benign lymph node involvement, respectively. The demographic data (age and gender) and ultrasonographic findings (shape, diameter, and number of lymph nodes) of the two groups were then compared.

\section{Statistical Analysis}

Data was analyzed by using SPSS statistical package version 20. Pearson's chi-squared test was used for comparison of categorical variables. For comparison of numerical data, Student's-t test was used. $\mathrm{P}<0.05$ was considered as statistically significant.

\section{Results}

Table 1. Comparison of the age and LAP diameter of the groups.

\begin{tabular}{llll}
\hline & $\begin{array}{l}\text { Group 1 }(\mathbf{n}=4) \\
\text { Mean }\end{array}$ & $\begin{array}{l}\text { Group 2 }(\mathbf{n}=19) \\
\text { Mean }\end{array}$ & $\boldsymbol{P}$ \\
\hline Age (years) & $55 \pm 22.33$ & $47.26 \pm 18.56$ & 0.63 \\
Diameter (mm) & $45 \pm 30.27$ & $26.2 \pm 15.68$ & 0.02 \\
\hline
\end{tabular}

$\mathrm{P}<0.05$ was considered as statistically significant.

Table 2. Comparison of the gender and US findings of the groups.

\begin{tabular}{llllll}
\hline & $\begin{array}{l}\text { Group 1 } \\
\text { N=4 }\end{array}$ & \% & $\begin{array}{l}\text { Group 2 } \\
\text { N=19 }\end{array}$ & \% & $\boldsymbol{P}$ \\
\hline $\begin{array}{l}\text { Gender } \\
\text { Female }\end{array}$ & 1 & 25 & 16 & 84.2 & NS \\
Male & 3 & 75 & 3 & 15.3 & 0.04 \\
US findings & & 75 & 2 & & \\
Malignancy & 3 & 25 & 17 & 89.5 & 0.04 \\
$\begin{array}{l}\text { Benign } \\
\text { Multiple LAP }\end{array}$ & 1 & 100 & 11 & 57.8 & NS \\
\hline
\end{tabular}

$\mathrm{P}<0.05$ was considered as statistically significant.

$N S$ : Non-significant

Seventeen $(73.91 \%)$ of the patients were female, and 6 $(26.09 \%)$ were male. The mean age of the patients was $46.6 \pm 18.94$ years. According to the pathology results, a malignant lymphoma was diagnosed in four patients in Group 1. Granulomatous lymphadenitis $(n=6)$ and reactive lymphadenitis $(n=13)$ were diagnosed in Group 2 . The mean age of the patients was 55 years in Group 1 and 47.26 in Group 2. There was no statistical difference between the groups in terms of age (Table 1). There were three male patients and one female patient in Group 1, and there were 16 female patients and three male patients in Group 2. There was a statistically significant between-group difference in gender, with a male gender predominance in the malignant lymphoma group $(p=0.04)$ (Table 2$)$. According to the ultrasonography (US) findings, the lymph node hilus was absent in $6(26.08 \%)$ patients, and the classical oval-shaped lymph node was round shaped in these patients. The classical oval shape and preservation of the lymph node hilus were observed in the other 17 patients. Based on the US findings, criteria for malignancy were observed in 3 (75\%) of the four patients who were diagnosed with a malignant lymphoma in group 1. In group 2, two cases of malignancy based on the US findings were diagnosed as granulomatous lymphadenitis according to the pathology results. Multiple enlarged lymph nodes were detected in $15(65.21 \%)$ of the patients, and a single enlarged lymph node was detected in the other 8 $(30.43 \%)$ patients. In Group 1, all the patients $(100 \%)$ had multiple enlarged lymph nodes, whereas only $11(57.8 \%)$ of the patients in Group 2 had multiple enlarged lymph nodes. There was no significant difference between the groups in the numbers of lymph nodes $(p=0.15)$. The mean overall lymph node diameter was $29.4 \mathrm{~mm}$ (range, 5-80 $\mathrm{mm}$ ). The mean lymph node diameter was $45 \mathrm{~mm}$ in Group 1, and it was 26.2 $\mathrm{mm}$ in Group 2. There was statistically significant betweengroup difference in the lymph node diameter $(p=0.02)$.

\section{Discussion}

In the clinical examination of patients admitted with axillary LAP, it is important to assess the size, shape, and relationship with surrounding structures and to determine whether supraclavicular, inguinal lymph nodes and breast masses on the same side. Clinical, radiological, and blood laboratory findings may provide preliminary information about the nature of axillary LAP. In daily practice, the initial assessment after the clinical assessment involves ultrasonographic imaging. US is a sensitive imaging technique for axillary LAP, and and it can help to distinguish benign or malign pathologies by revealing the structure of the lymph nodes In US imaging, normal lymph nodes have an oval shape and consist of a hypoechoic cortex outside and a hyperechoic medulla inside. In contrast, metastatic and lymphomatous lymph nodes have a round shape, with progressive disappearance in hilus echogenicity. In one study, hilar echogenicity was exhibited in $84-92 \%$ of benign lymph nodes. Other studies reported that $74-96 \%$ of malignant lymph nodes did not show hilar echogenicity [4-7]. Consistent with this study, we observed US findings of malignancy criteria in $75 \%$ of the patients in our study. The pathology reports of two patients who were diagnosed with malignancy according to the US findings revealed caseificated granulamotous lymphadenitis.

Based on US findings, fine needle aspiration (FNA) can be performed to detect the nature of axillary LAP. Several clinical studies have demonstrated the high diagnostic accuracy rate of FNA biopsies [8-10]. In our hospital, imaging-guided biopsies of the axillary lymph nodes cannot be performed. This is a limitation of the present study.

Studies of the relationship of age with malignant LAP reported that the malignancy rate increased with age $[11,12]$. Conversely, we did not observe any relationship between age and malignancy in our two patient groups. Furthermore, in the present study, the majority of the patients diagnosed with a malignant lymphoma were males. Consistent with our findings, Castillo et al. [13] and Wan et al. [14] reported a higher male/female ratio.

Many studies reported that the diameter of the lymph 
nodes was a decisive factor in a diagnosis of malignancy $[15,16]$. Similarly, we found that the average diameters of the lymph nodes of those diagnosed with a malignant lymphoma were larger than those diagnosed with a benign disease.

\section{Conclusions}

Although the small number of patients is a limitation of the present study, we found that US was valuable in the diagnosis of patients with axillary LAP. Our findings suggest that a histopathological examination is warranted in male patients with an axillary LAP larger than $2 \mathrm{~cm}$ determined by US.

\section{References}

[1] Park JE, Sohn YM, Kim EK (2013) Sonographic findings of axillary masses: what can be imaged in this space? J Ultrasound Med 32: 1261-1270.

[2] Singh S, Mishra AK, Tewari S, Kumar S (2013) Bilateral axillary masses mimicking as accessory breast with multiple fibroadenoma and bilateral gigantomastia in HIV-positive patient. BMJ Case Rep 2013.

[3] De Andrade JM, Marana HR, Sarmento Filho JM, Murta EF, Velludo MA, et al. (1996) Differential diagnosis of axillary masses. Tumori 82: 596-599.

[4] Verbanck J, Vandewiele I, De Winter H, Tytgat J, Van Aelst F, et al. (1997) Value of axillary ultrasonography and sonographically guided puncture of axillary nodes: a prospective study in 144 consecutive patients. J Clin Ultrasound 25: 53-56.

[5] Feu J, Tresserra F, Fabregas R, Navarro B, Grases PJ, et al. (1997) Metastatic breast carcinoma in axillary lymph nodes: in vitro US detection. Radiology 205: 831-835.

[6] Rubaltelli L, Proto E, Salmaso R, Bortoletto P, Candiani F, et al. (1990) Sonography of abnormal lymph nodes in vitro: correlation of sonographic and histologic findings. AJR Am J Roentgenol 155: 1241-1244.

[7] Hrazdira I, Krupova M, Kyselova H (2005) [Possibilities of ultrasonographic differentiation of neck and axillary lymphadenopathy]. Vnitr Lek 51: 1371-1374.

[8] Fanny ML, Beyam N, Gody JC, Zandanga G, Yango F, et al. (2012) Fine-needle aspiration for diagnosis of tuberculous lymphadenitis in children in Bangui, Central African Republic. BMC Pediatr 12: 191.

[9] Marti JL, Ayo D, Levine P, Hernandez O, Rescigno J, et al. (2012) Nonimage-guided fine needle aspiration biopsy of palpable axillary lymph nodes in breast cancer patients. Breast J 18: 3-7.

[10] Hirachand S, Lakhey M, Akhter J, Thapa B (2009) Evaluation of fine needle aspiration cytology of lymph nodes in Kathmandu Medical College, Teaching hospital. Kathmandu Univ Med J (KUMJ) 7: 139-142.

[11] Lee Y, Terry R, Lukes RJ (1980) Lymph node biopsy for diagnosis: a statistical study. J Surg Oncol 14: 53-60.

[12] Bazemore AW, Smucker DR (2002) Lymphadenopathy and malignancy. Am Fam Physician 66: 2103-2110.

[13] Castillo JJ, Winer ES, Olszewski AJ (2013) Population-based prognostic factors for survival in patients with Burkitt lymphoma: an analysis from the Surveillance, Epidemiology, and End Results database. Cancer 119: 3672-3679.

[14] Wan WL, Tian L, Wang J, Zhang W, Jing HM, et al. (2013) [Clinical characteristics and prognostic factors of 62 cases with Hodgkin lymphoma]. Zhonghua Xue Ye Xue Za Zhi 34: 618-621.

[15] Vassilakopoulos TP, Pangalis GA (2000) Application of a prediction rule to select which patients presenting with lymphadenopathy should undergo a lymph node biopsy. Medicine (Baltimore) 79: 338-347.

[16] Slap GB, Brooks JS, Schwartz JS (1984) When to perform biopsies of enlarged peripheral lymph nodes in young patients. JAMA 252: 1321-1326. 